\title{
Knowledge, attitude and practices among pregnant women about antenatal care, danger sign during pregnancy and adopting family planning method
}

\author{
Punyatoya Bej \\ ${ }^{1}$ Associate Professor, Dept. of Community Medicine, Dr Baba Saheb Ambedkar Medical College and Hospital, New Delhi, India \\ *Corresponding Author: \\ Email: punyatoya_bej@yahoo.co.in
}

\begin{abstract}
Introduction: The knowledge, attitude and practices (KAP) for antenatal check-up during pregnancy is a key indicator of health care facility in a community. The study was conducted in a district hospital in North Delhi of India about antenatal care among pregnant female.

Materials and Methods: Total 54 pregnant females were studied about KAP during antenatal care check-up in a tertiary care hospital.The study was cross sectional in design. They were asked questions while attending the outpatient clinic in the hospital.

Result: It was found that practices and positive attitude about antenatal care among pregnant females was adequate. Score for positive attitude was $93 \%$ and score for antenatal care practices was $62 \%$. Both are more than 50\%. But knowledge among them is not adequate; the score for knowledge about antenatal care was $17 \%$, which was less than $50 \%$. Hence periodic health education sessions have to be conducted by the doctors and health staffs. Ten pregnant females(18.5\%) had faced danger signs during their previous pregnancy, these danger signs were dizziness and fainting, persistent vomiting, less movement of baby inside uterus, bleeding per vagina (for abortion), persistent vomiting, leaking per vagina and pain abdomen. Four pregnant women $(7.4 \%$ ) had mentioned bleeding per vagina in their previous pregnancy. Out of 54 pregnant women nine pregnant female told that they were using condom and six not using any contraceptive methods after marriage.

Conclusion: The study detected knowledge score of $17 \%$, practice score of $62 \%$ and attitude score of $93 \%$ in pregnant female attending antenatal check-up clinic.
\end{abstract}

Keywords: Antenatal care, Family planning, Danger signs, Knowledge, Attitude, Practices.

\section{Introduction}

Antenatal care is the care of the women during pregnancy. The primary aim of antenatal care is to achieve a healthy mother and a healthy baby at the end of pregnancy. The objectives of antenatal care are to promote, protect and maintain the health of the mother during pregnancy. Antenatal care helps to detect high risk cases, to foresee complications and to remove anxiety and dread associated with delivery.

Knowledge is the understanding of any given topic. ${ }^{1}$ In this study, it refers to a pregnant women's understanding of components of antenatal care which include registration of pregnancy, danger signs during pregnancy, intake of prophylactic Iron and Folic Acid (IFA) tablets during pregnancy and adapting family planning methods.

Attitudes are emotional, motivational, perceptive and cognitive beliefs that positively or negatively influence the behaviour or practice of an individual. ${ }^{2} \mathrm{~A}$ pregnant female's antenatal check-up, adapting family planning behaviour is influenced by her emotions, motivations, perceptions and thoughts. Attitudes influence future behaviour no matter the individual's knowledge and help to explain why an individual adopts one practice and no other alternatives. The terms attitude, beliefs and perceptions are interchangeable.

Practices are defined as the observable actions of a pregnant women that could affect her to go to the hospital for antenatal check-up, after knowing the danger signs during pregnancy, how she is making the arrangement to attend hospital and how she had adapting the family planning methods after marriage, in the previous and present pregnancy.

With this background the present study was conducted among pregnant females to find out the knowledge, attitude and practice (KAP) score about antenatal care.

\section{Aims and Objectives}

1. To find out the percentage and KAP score of antenatal care

2. To find out the percentage and KAP score of danger signs during pregnancy

3. To find out the percentage and KAP score of adoption of family planning methods in women attending antenatal clinic.

\section{Material and Methods}

The study was conducted after institutional ethical committee approval and written informed consent from the participants. The study was designed as a cross sectional study. Study population were pregnant females attending antenatal clinic in a tertiary level hospital. Fifty four (54) pregnant females recruited randomly from the pregnant females visiting the tertiary level hospital in obstetrics and gynaecology department.

Sampling Technique: In this district level hospital antenatal check-up is scheduled every day. On an average 50 pregnant females attend outpatient department (OPD) antenatal clinic every day. So every 
day 3 pregnant females were asked question about KAP for antenatal care, danger sign during pregnancy and method of adopting family planning method after marriage and present pregnancy.

Data Collection: Women were questioned regarding registration of pregnancy, minimum 4 antenatal checkup visits, taking 100 iron and folic acid tablets, type of family planning method was adopted by them after marriage in case of nulliparous female and what is their knowledge to adopt the family planning method after the present pregnancy. They were also asked about the danger sign during pregnancy. The danger signs were dizziness, fainting swelling of feet, severe head ache, persistent vomiting, bleeding per vagina, and blurring of vision, less movement of baby inside uterus, leaking per vagina and pain abdomen.

Data Analysis: For correct answer for KAP score of 1 is given. While incorrect response was given 0 . Adequate KAP was defined as those getting a score of $50 \%$ and above of the total score and inadequate KAP as getting a score of less than $50 \%$ of the total score. Indicators used to quantify knowledge are reported in terms of percentages and score.

Percentage: Percentages used as indicators of knowledge are determined from the numerical indicators. For example: Percentage of respondents who know the correct answer to a question;

Score - For a score-based indicator of knowledge, each respondent is given a score based on the number of correct responses provided. The knowledge score of the population is calculated for each question as the total number of correct response to one question divided by the total number of responses. Respondents who did not answer the question, or for whom information is incomplete were excluded from the study.

Score of knowledge $=\frac{\text { Sum of correct responses given by all respondents }}{\text { Total number of respondents }}$

Measurement of attitudes- Attitudes are measured by asking the respondents to judge whether they are positively or negatively inclined towards:

1. Antenatal check up

2. Adopting family planning method

3. Danger signs during pregnancy

Questions are aimed at measuring attitudes were developed based on the Health Belief Model. ${ }^{1}$ According to this model, people's beliefs influence their health-related actions. ${ }^{2}$ It states that the likelihood that an individual will take action to prevent a health problem depends on the individual's perception of the condition's severity and his/ her likelihood of getting it, on the benefits of and barriers to taking action to reduce the risk of getting the condition and on his/her confidence in taking action.

Total 14 questions were asked about knowledge aspect of antenatal care, perceived danger signs during pregnancy and contraceptive uses. Then score for knowledge about antenatal care is calculated. Similarly the practices followed by the pregnant female regarding antenatal care, after facing danger signs during pregnancy and adapting family planning methods were asked. The questions regarding practices contain 14 questions. Then score for practices regarding antenatal care was calculated. Positive attitude about antenatal care contain eight questions. Then score for positive attitude about antenatal care was calculated.

\section{Result}

A total of 54 pregnant women completed the study. The demographic profile is depicted in Table 1.

The ANC practices of pregnant female are shown in Table 2. Ten pregnant females had faced danger signs during their previous pregnancy.Four pregnant women had mentioned bleeding per vagina in their previous pregnancy. The knowledge about antenatal care is shown in Table 3.

Fourteen pregnant females reported that they know about the danger signs during pregnancy. These pregnant females had mentioned the danger signs during pregnancy as severe abdominal pain, less fetal movement, any problem, bleeding per vagina, severe headache, vomiting, palpitation, white discharge and nausea. These danger signs required immediate consultation with doctor or health care providers.

Attitude among Pregnant Female about Antenatal Care: The pregnant females were asked about the benefit they are getting by attending antenatal care clinic. Maximum respondent $(n=20)$ told that they get admitted in the hospital for safe child birth. Then followed by 18 respondent told to get advice regarding danger signs during pregnancy. The pregnant female also respond that they can know about anaemia status, get advice from doctor to have safe child birth and have regular antenatal check-up, to have good health, to have healthy baby and mother and to have no illness. Nine pregnant females told that they come to the hospital to get free IFA (Iron \& folic acid) and calcium tablets. Twelve pregnant female tell they come to hospital to get tetanus toxoid injection and getting advice from doctors. Fifty one pregnant female tell that if they perceive any danger signs during pregnancy, they consult immediately to a doctor. Only few respond that they consult to a Accredited Social Health Activist (ASHA) worker. Forty pregnant females respond that after knowing the benefits of family planning methods, they will adopt the family planning methods. Few respond that, they have weak body, so can't adapt any method of family planning and some told that it is not necessary to adopt family planning methods. Few respond that they have 2 girls, for that reason not using any contraception method. Only one had given the respond that she had 1st girl child, 2nd not known, so can't take decision about adopting the family planning methods. Fifty two pregnant females responded that they had taken decision to give birth the baby in the hospital (Table 4). Only two pregnant females take 
decision to give child birth in the hospital by the advice of the health workers. Forty pregnant female take decision from both their husbands and other family members.

Score of knowledge about antenatal care, danger sign during pregnancy and adopting family planning methods was $17 \%$.

Score of knowledge $=\frac{3+0+45+20+0+0+4+1+8+11+34+14+1+7}{54 \times 14} \times 100=17 \%$

Score of practices about ante natal care, danger sign during pregnancy and adopting family planning methods was $62 \%$.

Score of positive attitude of antenatal care $=\frac{54+45+52+53+49+45+52+53}{54 \times 14} \times 100=93 \%$

Score of positive attitude about antenatal care, danger sign during pregnancy $\&$ adapting family planning methods was $93 \%$.

Score of positive attitude of antenatal $\mathrm{care}=\frac{54+45+52+53+49+45+52+53}{54 \times 14} \times 100=93 \%$

Table 1: Socio demographic profile of pregnant females attending antenatal care services

\begin{tabular}{|l|c|}
\hline \multicolumn{1}{|c|}{ Socio demographic parameters } & Numbers (Percentage) \\
\hline Age in year (<30 years) & $50(92.59 \%)$ \\
\hline Illiterate & $7(12.96 \%)$ \\
\hline Qualification below class 10 & $23(42.59 \%)$ \\
\hline Qualification of class 10 pass & $10(18.51 \%)$ \\
\hline Graduation pass & $10(18.51 \%)$ \\
\hline Post-graduation & $4(7.40 \%)$ \\
\hline Working (Job holder) & $3(5.55 \%)$ \\
\hline Staying in Joint family & $39(72.23 \%)$ \\
\hline $\begin{array}{l}\text { Gestation week of more than 37 weeks of pregnant women at } \\
\text { the time of KAP study }\end{array}$ & $47(87.03 \%)$ \\
\hline Primi gravida women at the time of KAP study & $22(40.74 \%)$ \\
\hline Living in rented house & $28(51.85 \%)$ \\
\hline Migrants & $2(3.70 \%)$ \\
\hline
\end{tabular}

Table 2: Practices of pregnant females on antenatal care

\begin{tabular}{|l|c|}
\hline \multicolumn{1}{|c|}{ Practices of pregnant females on antenatal care } & $\begin{array}{c}\text { Numbers } \\
\text { (Percentage) }\end{array}$ \\
\hline Registered during first trimester & $42(77.78 \%)$ \\
\hline Diagnosed pregnancy by using pregnancy diagnosis kit & $48(88.89 \%)$ \\
\hline Completed more than four antenatal care visits & $37(68.51 \%)$ \\
\hline Taken tetanus toxoid injection & $47(87.03 \%)$ \\
\hline Taken IFA (iron folic acid ) supplementation & $44(81.48 \%)$ \\
\hline Gastritis due to intake of IFA tablets & $09(16.67 \%)$ \\
\hline Faced danger signs in previous pregnancy & $10(18.51 \%)$ \\
\hline Use transport under Janani Suraksha Yojana & $02(3.70 \%)$ \\
\hline Not used any contraception after marriage & $44(81.48 \%)$ \\
\hline Got information about contraception from health workers & $09(16.67 \%)$ \\
\hline Have no information about contraception & $20(37.03 \%)$ \\
\hline Got contraception from governmental facility & $06(11.12 \%)$ \\
\hline Family members encourage to attend antenatal care visits & $30(55.56 \%)$ \\
\hline Using condom & $09(16.67 \%)$ \\
\hline Not using any contraceptive methods & $06(11.12 \%)$ \\
\hline
\end{tabular}


Table 3: Knowledge among pregnant female about antenatal care Knowledge among pregnant female about antenatal care Ideally 13 antenatal care visits to be made

\begin{tabular}{ll}
\hline Minimum four antenatal care visits to be made & 00
\end{tabular}

Registration of pregnancy to be done as early as possible when pregnancy is diagnosed

Body fitness and laboratory investigation to be done before pregnancy

Urine test, HIV test, sugar, Hbs Ag, thyroid, VDRL, Haemoglobin, thalassemia and blood test to be done before pregnancy

2 tetanus toxoid doses required to immunise against tetanus during pregnancy

Only iron and folic acid (IFA) tablets to be given prophylactically

IFA and calcium both to be taken during pregnancy

Don't know how many IFA tablets to be taken

100 IFA tablets to be given to mild anaemic pregnant female

Under JSY (Janani Suraksha Yojana) financial assistance is not provided for child birth

Financial assistance is provided under JSY

No transport provided under JSY to them to reach hospital for child birth

Anganwadi provide diet to pregnant female

Knowledge about the danger signs during pregnancy

Don't know which contraceptive method to be use after marriage

Don't know which type of family planning method to be practiced

after their present pregnancy

00

$03(5.5 \%)$

00

$20(37.03)$

$20(37.03 \%)$

$9(90.7 \%)$

$1(20.37 \%)$

$36(66.67 \%)$

$46(85.18 \%)$

$05(9.25 \%)$

$42(77.77 \%)$

$08(14.81 \%)$

$42(77.77 \%)$

$35(64.81 \%)$

$14(25.92 \%)$

$49(90.74 \%)$

$39(72.22 \%)$

Table 4: Attitude of pregnant female about antenatal care

\begin{tabular}{|l|c|c|c|}
\hline \multicolumn{1}{|c|}{ Questions asked } & \multicolumn{2}{c|}{ Numbers of respondents (\%) } \\
\hline $\begin{array}{l}\text { Necessary to register during pregnancy for } \\
\text { antenatal check - up }\end{array}$ & $50(92.59 \%)$ & 00 & $04(7.4 \%)$ \\
\hline $\begin{array}{l}\text { Necessary to know about danger signs during } \\
\text { pregnancy }\end{array}$ & $52(96.29)$ & $01(1.85 \%)$ & $01(1.85 \%)$ \\
\hline $\begin{array}{l}\text { Necessary to know about which family planning } \\
\text { method to adopt after marriage and after child } \\
\text { birth to limit the number of children }\end{array}$ & $49(90.74)$ & $04(7.40)$ & $01(1.85 \%)$ \\
\hline $\begin{array}{l}\text { Decision of respondent to give birth the baby in the } \\
\text { hospital }\end{array}$ & $52(96.29 \%)$ & 00 & $02(3.7 \%)$ \\
\hline
\end{tabular}

\section{Discussion}

The KAP (Knowledge, Attitude \& Practices) study was conducted in a district level hospital in North Delhi about ante natal care among pregnant female. It was found that practices and positive attitude about antenatal care among pregnant females was adequate. Score for positive attitude was $93 \%$ and score for antenatal care practices was $62 \%$. Both are more than $50 \%$. But knowledge among them was not adequate; the score was $17 \%$, which was less than $50 \%$. Most of the pregnant female comings to this hospital were from periphery dispensaries of Delhi. Most of them were referral patients. Patients were also coming for infertility treatment. They conceived after success of treatment. They were getting good advice from health workers to attend the antenatal clinic. Hence, they had followed adequate practices about antenatal care.

A study conducted by Laishram $J$ et al found that the mean score of the knowledge on antenatal care was $20.9 \pm 4.3$ out of a total score of 36 , which was $55.56 \% .^{3}$ It is quiet higher than our study. In their study the knowledge component does not include danger sign during pregnancy. In the same study $42.6 \%$ women got full antenatal care and main reason for not attending any antenatal check - up were thought of as not necessary and financial constraints. It was found that 
higher level of education, Hindu religion, age at marriage and living in owned house were statistically associated with better knowledge. Here full antenatal is define as at least 3 visits for antenatal check-up, at least one tetanus toxoid injection received and 100 IFA tablets/ syrup consumed. Question asked from women who delivered in last 5 years and residing in an urban area. ${ }^{3}$ But in our study the questions were asked to pregnant women for their knowledge, attitude and practices regarding antenatal care of their present pregnancy.

Chandhiok $\mathrm{N}$ et al conducted a study on antenatal care utilisation in different part of India found that $73.9 \%$ of women had completed 3 antenatal visits. ${ }^{4}$ In our study $68.5 \%$ pregnant female had completed four antenatal care visits. In another study at least one antenatal clinic visit were made by $97.4 \%$ of pregnant women. $^{3}$

A study done by Khatib $\mathrm{N}$ et al, in which the minimum 3 antenatal visits made by pregnant female is $33.6 \%{ }^{5}$ Iron tablets were taken by $94 \%$ of women, though only $45.3 \%$ completed the required 100 tablets. ${ }^{3}$ In our study 44 pregnant female (81.4\%) has taken IFA tablets.

A study conducted in Alexandria in which the total score for the knowledge on antenatal care in urban women was $11.23 \pm 2.91$ and in rural women the knowledge score was $6.59 \pm 4.14 .{ }^{6}$ In our study also the knowledge score is less and it is $17 \%$. The attitude score for antenatal care among urban women was $10.60 \pm 2.33$ and the attitude score for antenatal care among rural women was $8.55 \pm 2.39 .{ }^{6}$ But in our study about positive attitude towards antenatal care is $93 \%$. So the study conducted by Kishk NA in Alexandria the attitude among pregnant female and their family members regarding antenatal care is very less. About $45.3 \%$ of urban women had not been vaccinated against tetanus toxoid vaccine during their last pregnancy compared to only $24.7 \%$ of rural area. ${ }^{6}$ In our study forty seven $(87.03 \%)$ has taken tetanus toxoid injection.

A study by Ibrahim HK et al found that $85.3 \%$ of pregnant women had a high knowledge score regarding antenatal care but in our study the knowledge score is only $17 \%$ and most of them $(96.0 \%)$ showed a positive attitude and in our study also positive attitude is $93 \%$. The highest percentage $(76.4 \%)$ of pregnant women also had good practice scores. ${ }^{7}$ In our study the practice score is $62 \%$.

A study by Patel BB found that $58 \%$ women attending an antenatal clinic in a tertiary care hospital had adequate knowledge regarding antenatal care. In the same study $100 \%$ women had a positive attitude towards antenatal care and $70 \%$ women practicing antenatal care adequately. ${ }^{8}$ In this study knowledge was assessed about antenatal care visits, tetanus toxoid immunisation, investigations and nutritional factors, danger signs of pregnancy, contraception and personal habit. Here score $>70 \%$ is considered adequate and score $<70 \%$ is considered inadequate. ${ }^{8}$ But in our study adequate knowledge is taken when score is $>50 \%$. Variable to assess attitude were opinion on the place of child birth, the effect of smoking and alcohol on the health of mother and fetus, antenatal care registration, antenatal carevisits and motivation, investigation and dietary changes. ${ }^{8}$ Iron folic acid intake and regularity of intake also taken into consideration for assessing attitude of pregnant females and their family member. Questions were asked to assess the practiceswith regards to antenatal care visits, dietary changes made during pregnancy, iron and folic acid tablets intake, tetanus toxoid immunisation and use of contraception was also noted. Question related to smoking, alcohol and self-medication were noted. ${ }^{8}$ But in our study question related to dietary intake, smoking and alcohol were not taken.

A study by Gupta RK et al found that $10.9 \%$ of pregnant female knew that $>3$ antenatal care visits were essential. ${ }^{9}$ In our study, none of the pregnant female knew that minimum four antenatal care visits are necessary.Regarding the knowledge about antenatal care services $86.2 \%$ knew about early registration (preferably before 16 weeks) to be done to have proper antenatal care. $94.3 \%$ had adequate knowledge about the importance of tetanus toxoid injection during pregnancy. ${ }^{9}$ In our study forty nine $(90.7 \%)$ pregnant female responded that 2 tetanus toxoid doses required to immunise against tetanus during pregnancy.

The knowledge of antenatal care and its importance was known to $82.8 \%$ women. ${ }^{10}$ In our study $92.5 \%$ pregnant females responded that registration for antennal care and visits is essential. Shafqat $\mathrm{T}$ et al reported that $83.98 \%$ women had awareness regarding appropriate diet and $80.85 \%$ had knowledge regarding iron and vitamin supplementation during pregnancy. ${ }^{10}$ In our study 35 female responded that anganwadi provide diet to pregnant female.

Hospital as a place of delivery was favoured by $82.03 \%$ women while $17.96 \%$ women preferred to deliver at homes and private clinics. ${ }^{10}$ In our study 52 (96.3\%) pregnant females responded that they had taken decision to give birth the baby in the hospital.

A study by Athanase $\mathrm{G}$ et al, in which the questions asked to women about antenatal care services of their previous pregnancy. Twelve percentage of women had one visit of antenatal clinic and $54.0 \%$ were have 2 antenatal visits and $18.6 \%$ have 3 antenatal visit and $54.0 \%$ have 4 antenatal visit. ${ }^{11}$ In our study thirty seven $(68.5 \%)$ pregnant female had completed more than four antenatal care visits.Importance of using contraceptive methods was known by $52.8 \%$ of pregnant females in a study by Athanase $\mathrm{G}$ et al in a municipal area of Tanzania. ${ }^{11}$ In our study $90.7 \%$ of pregnant female responded that it is necessary to know about family planning methods.

The complications arises due to hypertension was not known by $39.8 \%$ of the women and also they don't 
know that women with high blood pressure will affect the fetal growth in pregnancy. ${ }^{11}$ In our study $25.92 \%$ pregnant females reported that they knew about the danger signs during pregnancy. These pregnant females had mentioned the danger signs during pregnancy as severe abdominal pain, less fetal movement, any problem, bleeding per vagina, severe headache, vomiting, palpitation, white discharge and nausea.

Athanase $\mathrm{G}$ et al reported that $74.2 \%$ of women have positive attitude that early antenatal registration is important and $61.8 \%$ of the women have positive attitude towards the use of oral contraceptive pills. ${ }^{11}$ In our study $92.5 \%$ pregnant females have positive attitude towards registration of pregnancy.

All the studies have some variation in KAP score in pregnant females. The score has not reached towards $100 \%$. The practices and attitudes are better in percentage than knowledge component. We have studied the knowledge about danger signs during pregnancy by the females and also the postpartum contraception methods.

The limitations of the present study were that it was conducted in a small number of pregnant female, the participants were from urban community and hospital based study.

To conclude, the study detected knowledge score of $17 \%$, practice score of $62 \%$ and attitude score of 93\% in pregnant female attending antenatal check-up clinic. The study recommends strengthening of health education by health care providers to improve the knowledge component of KAP score during pregnancy and post-partum period, and early reporting of danger signs.

\section{References}

1. Kaliyaperumal K. Guideline for conducting a knowledge, attitude and practice (KAP) study. Community Ophthalmol, 2004;4:7-9.

2. M. Andrien. Social communication in nutrition: A methodology for intervention. FAO; 1994.www.fao.org/docrep/T0807e/T0807e00.htm

3. Jalina Laishram, Usha Devi Thounaojam, Jina Panmei, Salona Mukhia, H. Sanayaima Devi. Knowledge and practice of antenatal care in an urban area. Indian Medical Gazette: March 2013;101-106.

4. Chandhiok N, Dhillon B.S., Kambo L, Saxena N.C. Determinants of antenatal care utilisation in rural areas of India. A cross - sectional study from 28 districts (An ICMR task force study). J Obstet Gynecol India. 2006;56:47-52.

5. Khatib N, Zhiruddin QS, Gaidhane AM et al. Predictors for antenatal services and pregnancy outcome in a rural area: A prospective study in Wardha district, India, Indian J Med Sci. 2009;63:436-44.

6. Kishk NA. Knowledge, attitude and practices of women towards antenatal care; rural - urban comparison. J Egypt publichealth assoc. 2002;77:479 -98.

7. Ibrahim HK, El Borgy MD, Mohammed HO. Knowledge, attitude and practices of pregnant women towards antenatal care in primary health care centres in Benghazi,
Libya. J Egypt public health association. $2014 \mathrm{Dec}$ : 89(3);119-26.

8. Patel BB, Gurmeet P, Sinaikar DR, Pandya KH, Mahen A, Singh N. Astudy on knowledge and practices of antenatal care among pregnant women attending antenatal clinic at a tertiary care hospital of Pune, Maharastra. Med J DY Patil Univ. 2016;9:354-62.

9. Gupta RK, Shore TN, Verma AK, Jan R. Knowledge regarding antenatal care services, its utilisation and delivery practices in mothers (aged 15 - 49 years) in a rural area of North India. Trop J Med Res, 2015;18:89 94.

10. Shafqat T, Fayaz S, Rahim R, Saima S. Knowledge and awareness regarding antenatal care and delivery among pregnant women. J. Med. Sci. (Peshwar, print) 2015; 23:88-91.

11. Lilungulu AG, Matovelo D, Gesase A (2016) Reported Knowledge, Attitude and Practice of Antenatal Care Services among Women in Dodoma Municipal, Tanzania. $J$ Pediatr Neonatal Care 2016;4:00125. DOI: 10.15406/jpnc.2016.04.00125 